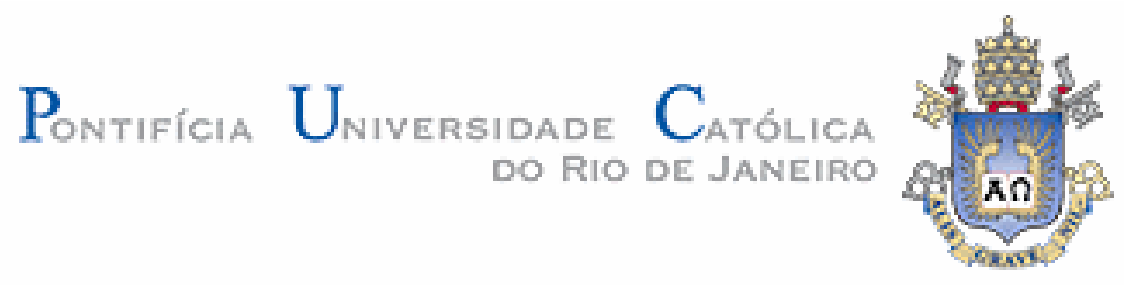

João Emido Lima da Silva Junior

\title{
Escoamento de Líquido Pseudo-Plástico Através de Capilar com Garganta
}

\begin{abstract}
Dissertação de Mestrado
Dissertação apresentada como requisito parcial para obtenção do título de Mestre pelo Programa de Pós-Graduação em Engenharia Mecânica da PUC-Rio.
\end{abstract}

Orientador: Prof. Márcio da Silveira Carvalho

Rio de janeiro Novembro de 2007 


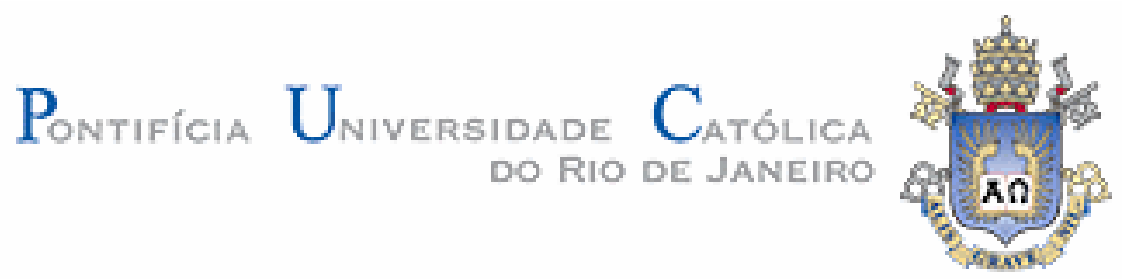

João Emido Lima da Silva Junior

\section{Escoamento de Líquido Pseudo-Plástico Através de Capilar com Garganta}

Dissertação apresentada como requisito parcial para obtenção do título de Mestre pelo Programa de PósGraduação em Engenharia Mecânica da PUC-Rio. Aprovada pela Comissão Examinadora abaixo assinada.

Prof. Márcio da Silveira Carvalho

Orientador

Departamento de Engenharia Mecância - PUC-Rio

Profa. Angela Ourivio Nieckele

Departamento de Engenharia Mecância - PUC-Rio

Prof. Oldrich Joel Romero Guzmán

Departamento de Engenharia Mecância - UFES

José Eugênio Leal

Coordenador Setorial do Centro

Técnico Científico - PUC-Rio

Rio de Janeiro, 26 de novembro de 2007 
Todos os direitos reservados. É proibida a reprodução total ou parcial do trabalho sem autorização da universidade, do autor e do orientador.

\section{João Emido Lima da Silva Junior}

Graduou-se em Engenharia Mecânica e Engenharia de Produção Mecânica na Pontifícia Universidade do Rio de Janeiro (Rio de Janeiro, Brasil).

Ficha Catalográfica

Silva Junior, João Emidio Lima da

Escoamento de líquido pseudo-plástico através de capilar com garganta / João Emidio Lima da Silva Junior; orientador: Márcio da Silveira Carvalho. -2007.

97 f. : il. ; $30 \mathrm{~cm}$

Dissertação (Mestrado em Engenharia Mecânica)-Pontifícia Universidade Católica do Rio de Janeiro, Rio de Janeiro, 2007.

Inclui bibliografia

1. Engenharia mecânica - Teses. 2. Fluido pseudo-plástico. 3. Capilar. 4. Meios porosos. I. Carvalho, Márcio da Silveira. II. Pontifícia Universidade Católica do Rio de Janeiro. Departamento de Engenharia Mecânica. III. Título.

CDD: 621 


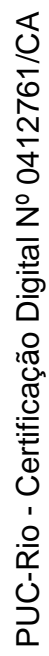

Para minha família e amigos. 


\section{Agradecimentos}

Ao meu orientador, Professor Márcio da Silveira Carvalho pela confiança e dedicação.

Aos professores do Departamento de Engenharia Mecânica da PUC-Rio pelos ensinamentos que ajudaram a construir a minha formação.

Aos Professores membros da comissão examinadora pelas sugestões e comentários que lapidaram o trabalho apresentado.

Ao meu pai, o Engenheiro João Emidio Lima da Silva pelo apoio, incentivo e dedicação incondicionais.

À minha mãe, a Sra. Marilda Cruz Lima da Silva, às minhas irmãs, Priscila Cruz Lima da Silva e Laura Cruz Lima da Silva e à minha namorada Isabel Sfoggia pela torcida e incentivo constantes.

Aos amigos, principalmente, Pedro Sabóia, Bruno Campos Machado, Bruno Alvarez de Azevedo Gomes e Frank Chaviano Pruzaesky, pelo grande incentivo e bons conselhos nos momentos difíceis.

Aos meus chefes na Companhia Brasileira de Petróleo Ipiranga, Flávio Dantas (anterior), Bismark Marco Duarte (Atual) e Mauro Umbelino (Atual), por terem tornado possível conciliar as obrigações profissionais com este mestrado.

Finalmente à PUC-Rio pela oportunidade oferecida. 


\section{Resumo}

Silva Junior, João Emidio Lima da; Carvalho, Márcio da Silveira. Escoamento de Líquido Pseudo-Plástico Através de Capilar com Garganta. Rio de janeiro, 2007. 97p. Dissertação de Mestrado Departamento de Engenharia Mecânica, Pontifícia Universidade Católica do Rio de Janeiro.

Devido às suas aplicações nas mais diversas áreas do conhecimento humano e em atividades vitais para o mundo moderno, como a prospecção e produção de petróleo, o escoamento de fluidos em meios porosos tem sido objeto de inúmeros estudos experimentais e teóricos. Por exemplo, na recuperação de campos de petróleo, depara-se com o desafio de prever o comportamento de fluidos a serem injetados na formação (gás, água ou água com adição de polímero), com o objetivo de aumentar a pressão e otimizar a recuperação de óleo. A abordagem adequada desta questão, implica na necessidade de profundo conhecimento do meio poroso e do comportamento dos fluidos envolvidos, bem como na utilização de modelos experimentais e/ou teóricos que propiciem simulações desses processos com razoável fidelidade. Neste trabalho o trajeto do fluido entre os espaços vazios de um meio poroso é representado por um capilar com contração/expansão (garganta). A partir de relações geométricas convenientemente definidas, onde apenas um parâmetro varia, foram geradas oito diferentes geometrias de análise. Foram feitas simulações numéricas de fluido newtoniano e pseudo-plástico utilizando-se código CFD desenvolvido na PUC-Rio. São estabelecidas relações entre o escoamento em capilares com garganta e o escoamento em capilares equivalentes, com raio constante. São apresentadas e comparadas duas metodologias, determinando raios equivalentes analíticos e ajustados $\left(R_{e q \text { an }}\right.$ e $R_{e q}$ aj). Para fluidos newtonianos, a aproximação é muito boa em todas as geometrias, sendo a maior diferença 6,2\%. Para fluido "power law" a diferença também é satisfatória, embora maior.

\section{Palavras-chave}

Fluido pseudo-plástico; capilar; meios porosos. 


\section{Abstract}

Silva Junior, João Emidio Lima da; Carvalho, Márcio da Silveira (advisor). Flow of pseudo-plastic liquid through capillary tubes with throat. Rio de janeiro, 2007. 97p. MSc. Dissertation - Departamento de Engenharia Mecânica, Pontifícia Universidade Católica do Rio de Janeiro.

Due to its application on a large number of fields and activities which are vital for the modern world, being oil prospection one of them, the flow of fluids on porous media have been studied in many experimental works and theoretical pieces. For instance, regarding the recovery of oil fields, it is challenging to predict the behavior of fluids that will be injected on the formation (gas, water or water added with polymer), with the objective to increase the pressure and optimize the oil recuperation. To approach this matter adequately a deep knowledge of the porous media and the behavior of the fluid in question are required, in addition to the use of the experimental and/or theorical models which provide simulations of this process with reasonable fidelity. On this experiment the path of the fluid through the empty spaces of a porous medium is represented by a capillary with contraction/expansion (throat). Starting with the conveniently defined geometrical relations where only one parameter varies, we generated eight different analysis geometries. Numerical simulations of the Newtonian and Pseudo-Plastic fluids were done using the CFD code developed at PUC-Rio. Relationships were established between the flow on capillaries with throat and equivalent capillaries, with constant radius. Two methodologies are presented and compared, determining equivalent analytical radius and adjusted

$\left(R_{e q a n}\right.$ e $\left.R_{e q a j}\right)$. For the case of Newtonians fluids, the approximation works very well on all geometries, and the largest difference is $6.2 \%$. Although larger, the approximation still works in a satisfactory manner when dealing with Power Law fluids.

\section{Keywords}

Pseudo-plastic fluid, capillary, porous media. 


\section{Sumário}

1 Introdução 18

1.1. Motivação do Trabalho 18

1.2. Descrição do Problema 21

1.3. Comportamento Mecânico dos Fluidos 23

1.3.1. Fluidos Newtonianos 23

1.3.2. Fluidos não-Newtonianos 23

1.4. Meios Porosos 25

1.5. Redes Capilares 27

1.6. Revisão da Literatura 29

1.7. Objetivos do Trabalho $\quad 35$

1.8. Roteiro 35

2 Formulação Matemática e Modelagem Computacional 37

2.1. Formulação Matemática $\quad 37$

2.1.1. Conservação de Massa

2.1.2. Conservação de Quantidade de Movimento Linear 38

2.1.3. Equações Constitutivas: Modelo "Power Law" e Carreau-Yasuda 39

2.1.4. Parâmetros Adimensionais 40

2.2. Geometria de Análise 40

2.3. Modelagem Computacional 43

2.3.1. Condições de Contorno 45

2.3.2. Solução do Sistema de Equações Diferenciais 46

3 Apresentação dos Resultados e Discussão 48

3.1. Teste de Malha 48

3.2. Raios Equivalentes

3.2.1. Fluido Newtoniano 56

3.2.2. Fluidos "Power Law"

3.3. Escoamento de Fluido Carreau-Yasuda 82

4 Comentários Finais $\quad 87$ 
Apêndice A - Cálculo da Geometria de Estudo 


\section{Lista de figuras}

Figura 1.1 - Representação esquemática do processo de recuperação de óleo por injeção de fluido em um meio poroso.

Figura 1.2 - Representação esquemática de meio poroso: (a) meio poroso e modelo de canal com constrição, (b) ilustração de rede de canais com constrições.

Figura 1.3 - Representação esquemática de canal convergente/divergente.

Figura 1.4 - Classificação do comportamento reológico de diferentes tipos de fluidos segundo Kawatra e Bakshi (Kawatra e Bakshi, 1996)

Figura 1.5 - Representação esquemática de meios porosos: (a) granular e (b) fibroso.

Figura 1.6 - Representação esquemática de uma rede de capilares.

Figura 1.7 Processo de geração de rede para arenito (Lopez et al., 2003): (a) imagem tridimensional do meio poroso obtida por simulação dos processos sedimentares pelos quais a rocha é formada, (b) Rede topologicamente equivalente de poros conectados por capilares.

Figura 1.8 - Diagrama esquemático da geometria de contração/expansão apresentada nos experimentos de Rothstein e McKinley (Rothstein e Mckinley, 2001).

Figura 2.1 - llustração tridimensional da geometria de análise.

Figura 2.2 - Superfície plana utilizada para formar a geometria de análise.

Figura 2.3 - Superfície plana utilizada para formar a geometria de análise, com as cotas básicas definidas.

Figura 2.4 - (a) representação física da geometria de estudo, (b) domínio físico de interesse, (c) Domínio físico de interesse dividido em regiões.

Figura 2.5 - Discretização da malha aplicada ao domínio físico.

Figura 2.6 - Condições de contorno.

Figura 3.1 - llustração das 3 diferentes malhas aplicadas à geometria 1 $\left(r_{o}=0,1\right)$ : (a) malha1 (grossa), (b) malha 2 (intermediária) e (c) malha 3 (fina).

Figura 3.2 - Teste de malha na geometria 1 ( $r_{o}=0,1$ u.c.) em escoamento de fluido newtoniano com $\mu=1.000$ Pa.s e $\rho=1.000 \mathrm{~kg} / \mathrm{m}^{3}$. 
Figura 3.3 - Teste de malha na geometria 1 ( $r_{o}=0,1$ u.c.) em escoamento do fluido de Carreau-Yasuda com $\eta_{0}=1.000$ Pa.s; $\eta_{\infty}=1$ Pa.s; $\lambda=0,1 ; n=0,6$ e $\rho=1.000 \mathrm{~kg} / \mathrm{m}^{3}$.

Figura 3.4 - Campo de pressões na geometria $1\left(r_{o}=0,1 \mathrm{~mm}\right)$ e linhas de corrente em escoamento de fluido newtoniano ( $\mu=1.000$ Pa.s e $\rho=$ $1.000 \mathrm{~kg} / \mathrm{m}^{3}$ ) e com $\Delta P$ total $(x$ de 0 a $12 \mathrm{~mm})=1,48 \times 10^{5} \mathrm{~Pa}$ : (a) Malha 1, (b) Malha 2 e (c) Malha 3.

Figura 3.5 - Campo de pressões na geometria $1\left(r_{o}=0,1 \mathrm{~mm}\right)$ e linhas de corrente em escoamento do fluido de Carreau-Yasuda $\left(\eta_{0}=1.000\right.$ Pa.s; $\eta_{\infty}=1$ Pa.s; $\lambda=0,1 ; n=0,6$ e $\rho=1.000 \mathrm{~kg} / \mathrm{m}^{3}$ ) e com $\Delta P$ total $(x$ de 0 a 12 $\mathrm{mm})=1,48 \times 10^{5} \mathrm{~Pa}:$ (a) Malha 1, (b) Malha 2 e (c) Malha 3.

Figura 3.6 - Pressão na linha de centro de escoamento na geometria 1 $\left(r_{o}=0,1 \mathrm{~mm}\right)$ em escoamento de fluido newtoniano ( $\mu=1.000$ Pa.s e $\rho=$ $1.000 \mathrm{~kg} / \mathrm{m}^{3}$ ) e com $\Delta P$ total $(x$ de 0 a $12 \mathrm{~mm})=1,48 \times 10^{5} \mathrm{~Pa}$.

Figura 3.7 - Pressão na linha de centro de escoamento na geometria 1 $\left(r_{o}=0,1 \mathrm{~mm}\right)$ em escoamento do fluido de Carreau-Yasuda $\left(\eta_{0}=1.000\right.$ Pa.s; $\eta_{\infty}=1$ Pa.s; $\lambda=0,1 ; n=0,6$ e $\left.\rho=1.000 \mathrm{~kg} / \mathrm{m}^{3}\right)$ e com $\Delta P$ total $(x$ de 0 a $12 \mathrm{~mm})=1,48 \times 10^{5} \mathrm{~Pa}$.

Figura 3.8 - Representação de canal convergente/divergente associado a canal com raio constante equivalente.

Figura 3.9 - Ilustração da geometria de análise com indicação de $\Delta P^{\prime}\left(P 2^{\prime}\right.$ $\left.P l^{\prime}\right)$ e dos trechos de entrada, da garganta e de saída do escoamento.

Figura 3.10 - Escoamento de fluido newtoniano ( $\mu=1.000$ Pa.s, $\rho=1.000$ $\left.\mathrm{kg} / \mathrm{m}^{3}\right)$ na geometria $1\left(r_{o}=0,1 \mathrm{~mm}\right)$, com $\Delta P$ total ( $x$ de 0 a $\left.12 \mathrm{~mm}\right) 1,5 \mathrm{x}$ $10^{5} \mathrm{~Pa}$ : (a) pressão na linha de centro, (b) Campo de pressões e linhas de corrente no trecho da garganta.

Figura 3.11 - Escoamento de fluido newtoniano ( $\mu=1.000$ Pa.s, $\rho=1.000$ $\left.\mathrm{kg} / \mathrm{m}^{3}\right)$ na geometria $2\left(r_{o}=0,2 \mathrm{~mm}\right)$, com $\Delta P$ total ( $x$ de 0 a $\left.12 \mathrm{~mm}\right) 1,5 \mathrm{x}$ $10^{5} \mathrm{~Pa}$ : (a) pressão na linha de centro, (b) Campo de pressões e linhas de corrente no trecho da garganta.

Figura 3.12 - Escoamento de fluido newtoniano ( $\mu=1.000$ Pa.s, $\rho=1.000$ $\left.\mathrm{kg} / \mathrm{m}^{3}\right)$ na geometria $3\left(r_{o}=0,3 \mathrm{~mm}\right)$, com $\Delta P$ total ( $x$ de 0 a $\left.12 \mathrm{~mm}\right) 1,5 \mathrm{x}$ $10^{5} \mathrm{~Pa}$ : (a) pressão na linha de centro, (b) Campo de pressões e linhas de corrente no trecho da garganta.

Figura 3.13 - Escoamento de fluido newtoniano ( $\mu=1.000$ Pa.s, $\rho=1.000$ 
$\left.\mathrm{kg} / \mathrm{m}^{3}\right)$ na geometria $4\left(r_{o}=0,4 \mathrm{~mm}\right)$, com $\Delta P$ total ( $x$ de 0 a $\left.12 \mathrm{~mm}\right) 1,5 \mathrm{x}$ $10^{5} \mathrm{~Pa}$ : (a) pressão na linha de centro, (b) Campo de pressões e linhas de corrente no trecho da garganta.

Figura 3.14 - Escoamento de fluido newtoniano ( $\mu=1.000$ Pa.s, $\rho=1.000$ $\left.\mathrm{kg} / \mathrm{m}^{3}\right)$ na geometria $5\left(r_{o}=0,5 \mathrm{~mm}\right)$, com $\Delta P$ total $(x$ de 0 a $12 \mathrm{~mm}) 1,5 \mathrm{x}$ $10^{5} \mathrm{~Pa}$ : (a) pressão na linha de centro, (b) Campo de pressões e linhas de corrente no trecho da garganta.

Figura 3.15 - Escoamento de fluido newtoniano ( $\mu=1.000$ Pa.s, $\rho=1.000$ $\left.\mathrm{kg} / \mathrm{m}^{3}\right)$ na geometria $6\left(r_{o}=0,6 \mathrm{~mm}\right)$, com $\Delta P$ total $(x$ de 0 a $12 \mathrm{~mm}) 1,5 \mathrm{x}$ $10^{5} \mathrm{~Pa}$ : (a) pressão na linha de centro, (b) Campo de pressões e linhas de corrente no trecho da garganta.

Figura 3.16 - Escoamento de fluido newtoniano ( $\mu=1.000$ Pa.s, $\rho=1.000$ $\left.\mathrm{kg} / \mathrm{m}^{3}\right)$ na geometria $7\left(r_{o}=0,7 \mathrm{~mm}\right)$, com $\Delta P$ total $(x$ de 0 a $12 \mathrm{~mm}) 1,5 \mathrm{x}$ $10^{5} \mathrm{~Pa}$ : (a) pressão na linha de centro, (b) Campo de pressões e linhas de corrente no trecho da garganta.

Figura 3.17 - Escoamento de fluido newtoniano ( $\mu=1.000$ Pa.s, $\rho=1.000$ $\left.\mathrm{kg} / \mathrm{m}^{3}\right)$ na geometria $8\left(r_{o}=0,8 \mathrm{~mm}\right)$, com $\Delta P$ total $(x$ de 0 a $12 \mathrm{~mm}) 1,5 \mathrm{x}$ $10^{5} \mathrm{~Pa}$ : (a) pressão na linha de centro, (b) Campo de pressões e linhas de corrente no trecho da garganta.

Figura 3.18 - Campo de pressões na geometria $1\left(r_{o}=0,1 \mathrm{~mm}\right)$ e linhas de corrente em escoamento do fluido de "power law" ( $m=1.000$ Pa.s, $n=0,8$ e $\rho=1.000 \mathrm{~kg} / \mathrm{m}^{3}$ ): (a) $\Delta P=3,0 \times 10^{4} \mathrm{~Pa}$, (b) $\Delta P=1,5 \times 10^{5} \mathrm{~Pa}$, (c) $\Delta P=3,0$ $\times 10^{5} \mathrm{~Pa}$.

Figura 3.19 - Campo de pressões na geometria $5\left(r_{o}=0,5 \mathrm{~mm}\right)$ e linhas de corrente em escoamento do fluido de "power law" ( $m=1.000$ Pa.s, $n=0,8$ e $\rho=1.000 \mathrm{~kg} / \mathrm{m}^{3}$ ): (a) $\Delta P=3,0 \times 10^{4} \mathrm{~Pa}$, (b) $\Delta P=1,5 \times 10^{5} \mathrm{~Pa}$, (c) $\Delta P=3,0$ $\times 10^{5} \mathrm{~Pa}$.

Figura 3.20 - Campo de pressões na geometria $8\left(r_{o}=0,8 \mathrm{~mm}\right)$ e linhas de corrente em escoamento do fluido de "power law" ( $m=1.000$ Pa.s, $n=0,8$ e $\rho=1.000 \mathrm{~kg} / \mathrm{m}^{3}$ ): (a) $\Delta P=3,0 \times 10^{4} \mathrm{~Pa}$, (b) $\Delta P=1,5 \times 10^{5} \mathrm{~Pa}$, (c) $\Delta P=3,0$ $\times 10^{5} \mathrm{~Pa}$.

Figura 3.21 - Campo de pressões na geometria $5\left(r_{o}=0,5 \mathrm{~mm}\right)$ e linhas de corrente em escoamento do fluido de "power law" ( $m=1.000$ Pa.s, $n=0,7$ e $\rho=1.000 \mathrm{~kg} / \mathrm{m}^{3}$ ): (a) $\Delta P=3,0 \times 10^{4} \mathrm{~Pa}$, (b) $\Delta P=1,5 \times 10^{5} \mathrm{~Pa}$, (c) $\Delta P=3,0$ $\times 10^{5} \mathrm{~Pa}$.

Figura 3.22 - Pressão na linha de simetria em escoamentos de fluido de 
"power law" ( $m=1.000$ Pa.s; $n=0,8$; e $\rho=1.000 \mathrm{~kg} / \mathrm{m}^{3}$ ) com $\Delta P=3,0 \mathrm{x}$ $10^{4}$, nas geometrias 1,5 e 8.

Figura 3.23 - Pressão na linha de simetria em escoamentos de fluido de "power law" ( $m=1.000$ Pa.s; $n=0,8$; e $\rho=1.000 \mathrm{~kg} / \mathrm{m}^{3}$ ) com $\Delta P=1,5 \mathrm{x}$ $10^{5}$, nas geometrias 1,5 e 8.

Figura 3.24 - Pressão na linha de simetria em escoamentos de fluido de "power law" ( $m=1.000$ Pa.s; $n=0,8$; e $\rho=1.000 \mathrm{~kg} / \mathrm{m}^{3}$ ) com $\Delta P=3,0 \mathrm{x}$ $10^{5}$, nas geometrias 1,5 e 8.

Figura 3.25 - Fluido de Carreau-Yasuda variando do expoente $n\left(\eta_{0}=1.000\right.$ $\mathrm{Pa} ; \eta_{\infty}=1 \mathrm{~Pa} ; \lambda=0,1$ e $\left.\rho=1.000 \mathrm{~kg} / \mathrm{m}^{3}\right)$, através da geometria $1\left(\underline{r}_{o}=0,1\right.$ $\mathrm{mm})$

Figura 3.26 - Fluido de Carreau-Yasuda variando do expoente $n\left(\eta_{0}=1.000\right.$ $\mathrm{Pa} ; \eta_{\infty}=1 \mathrm{~Pa} ; \lambda=0,1$ e $\left.\rho=1.000 \mathrm{~kg} / \mathrm{m}^{3}\right)$, através da geometria $5\left(r_{o}=0,5\right.$ $\mathrm{mm})$

Figura 3.27 - Fluido de Carreau-Yasuda variando do expoente $n\left(\eta_{0}=1.000\right.$ $\mathrm{Pa} ; \eta_{\infty}=1 \mathrm{~Pa} ; \lambda=0,1$ e $\left.\rho=1.000 \mathrm{~kg} / \mathrm{m}^{3}\right)$, através da geometria $8\left(r_{o}=0,8\right.$ $\mathrm{mm})$

Figura 3.28 - Fluido de Carreau-Yasuda variando o tempo de relaxação $\lambda$ $\left(\eta_{0}=1.000 \mathrm{~Pa} ; \eta_{\infty}=1 \mathrm{~Pa} ; n=0,7\right.$ e $\left.\rho=1.000 \mathrm{~kg} / \mathrm{m}^{3}\right)$, através da geometria 1 $\left(r_{o}=0,1 \mathrm{~mm}\right)$

Figura 3.29 - Fluido de Carreau-Yasuda variando o tempo de relaxação $\lambda$ $\left(\eta_{0}=1.000 \mathrm{~Pa} ; \eta_{\infty}=1 \mathrm{~Pa} ; n=0,7\right.$ e $\left.\rho=1.000 \mathrm{~kg} / \mathrm{m}^{3}\right)$, através da geometria 3 $\left(r_{o}=0,5 \mathrm{~mm}\right.$. $)$

Figura 3.30 - Fluido de Carreau-Yasuda variando o tempo de relaxação $\lambda$ $\left(\eta_{0}=1.000 \mathrm{~Pa} ; \eta_{\infty}=1 \mathrm{~Pa} ; n=0,7\right.$ e $\left.\rho=1.000 \mathrm{~kg} / \mathrm{m}^{3}\right)$, através da geometria 4 $\left(r_{o}=0,8 \mathrm{~mm}\right.$.)

Figura A.1 - Figura geométrica utilizada para cálculo das medidas necessárias à definição da geometria de estudo.

Figura A.2 - Geometria com $r_{o}=0,1 \mathrm{~mm}$ (geometria 1).

Figura A.3 - Geometria com $r_{o}=0,2 \mathrm{~mm}$ (geometria 2).

Figura A.4 - Geometria com $r_{o}=0,3 \mathrm{~mm}$ (geometria 3).

Figura A.5 - Geometria com $r_{o}=0,4 \mathrm{~mm}$ (geometria 4).

Figura A.6 - Geometria com $r_{o}=0,5 \mathrm{~mm}$ (geometria 5).

Figura A.7 - Geometria com $r_{o}=0,6 \mathrm{~mm}$ (geometria 6).

Figura A.8 - Geometria com $r_{o}=0,7 \mathrm{~mm}$ (geometria 7). 


\section{Lista de tabelas}

Tabela 2.1 - Medidas da geometria de estudo 43

Tabela 3.1 - Resumo da malha $1 \quad 48$

Tabela 3.2 - Resumo da malha $2 \quad 48$

Tabela 3.3 - Resumo da malha $3 \quad 49$

Tabela 3.4 - Resultados de fluido newtoniano ( $\mu=1.000$ Pa.s e $\rho=1.000$ $\left.\mathrm{kg} / \mathrm{m}^{3}\right)$ na geometria $1\left(r_{o}=0,1 \mathrm{~mm}\right) \quad 58$

Tabela 3.5 - Resultados de fluido newtoniano ( $\mu=1.000$ Pa.s e $\rho=1.000$ $\left.\mathrm{kg} / \mathrm{m}^{3}\right)$ na geometria $5\left(r_{o}=0,5 \mathrm{~mm}\right) \quad 58$

Tabela 3.6 - Resultados de fluido newtoniano ( $\mu=1.000$ Pa.s e $\rho=1.000$ $\left.\mathrm{kg} / \mathrm{m}^{3}\right)$ na geometria $8\left(r_{o}=0,8 \mathrm{~mm}\right) . \quad 59$

Tabela 3.7 - Comparação de $R_{e q a j}$ e $R_{\text {eq an }}$ - fluido newtoniano ( $\mu=1.000$ Pa.s e $\rho=1.000 \mathrm{~kg} / \mathrm{m}^{3}$ ).

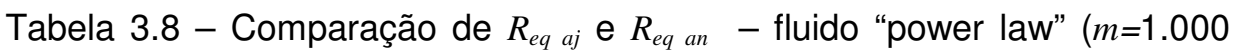
Pa.s, $n=0,8$ e $\left.\rho=1.000 \mathrm{~kg} / \mathrm{m}^{3}\right)$ na geometria $1\left(r_{o}=0,1 \mathrm{~mm}\right)$.

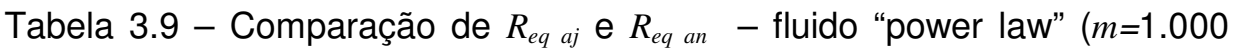
Pa.s, $n=0,8$ e $\left.\rho=1.000 \mathrm{~kg} / \mathrm{m}^{3}\right)$ na geometria $5\left(r_{o}=0,5 \mathrm{~mm}\right)$.

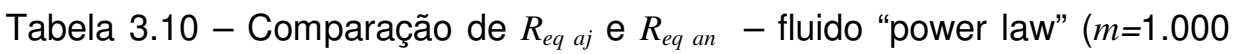
Pa.s, $n=0,8$ e $\left.\rho=1.000 \mathrm{~kg} / \mathrm{m}^{3}\right)$ na geometria $8\left(r_{o}=0,8 \mathrm{~mm}\right)$.

Tabela 3.11 - Comparação de $R_{e q ~ a j}$ e $R_{e q ~ a n ~}$ - fluido "power law" ( $m=1.000$ Pa.s, $n=0,7$ e $\left.\rho=1.000 \mathrm{~kg} / \mathrm{m}^{3}\right)$ na geometria $5\left(r_{o}=0,5 \mathrm{~mm}\right)$ 


\section{Lista de Símbolos}

\section{Símbolos Romanos}

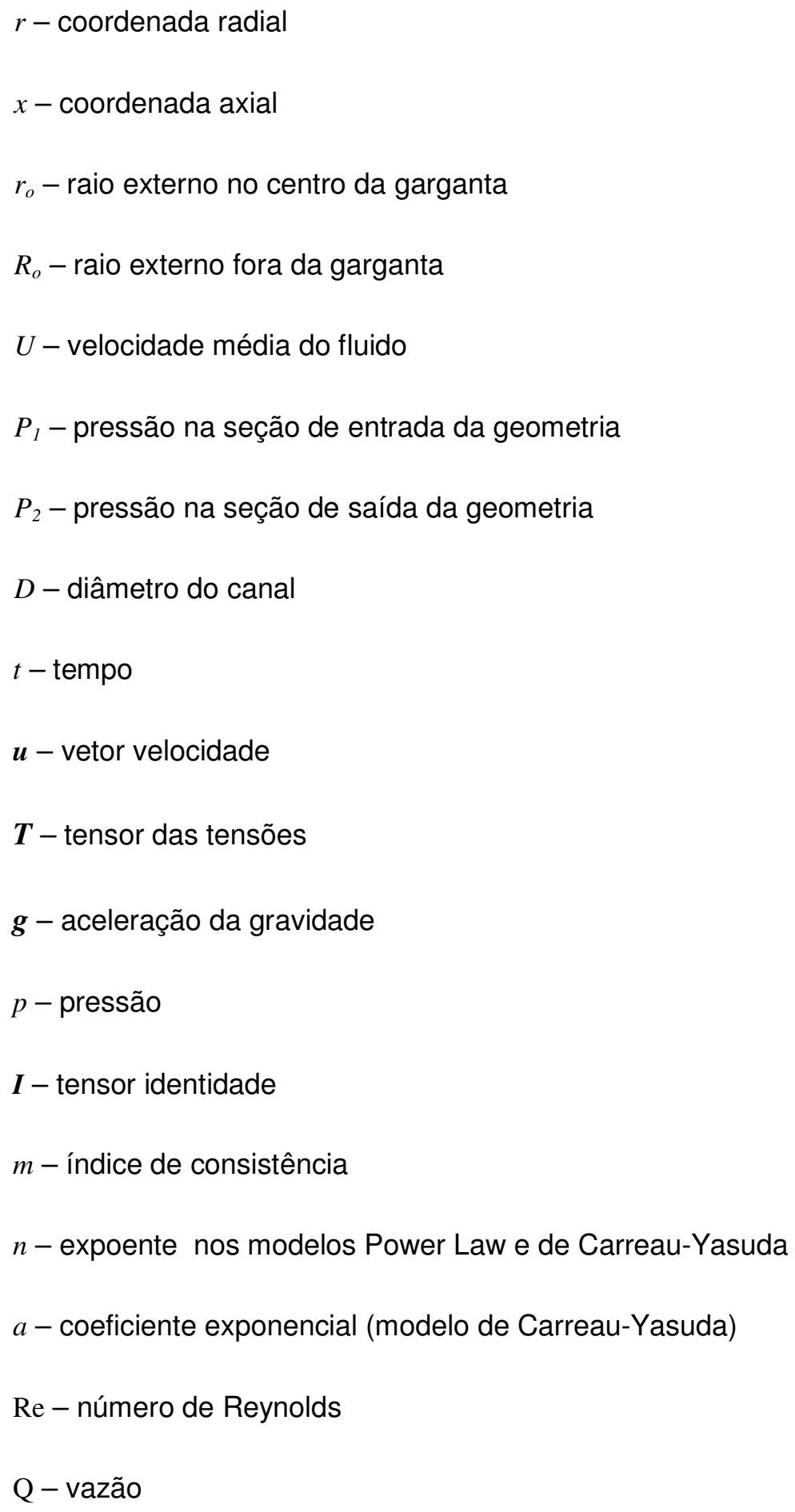


$d_{0}$ - diâmetro externo no centro da garganta

$\Delta P^{*}$ - variação adimensional de pressão

$\Delta P$ - variação de pressão no capilar

$\Delta P^{\prime}$ - diferença de pressão entre a saída e a entrada da garganta

$P_{2}^{\prime}$ - pressão na saída da garganta

$P_{l}^{\prime}$ - pressão na entrada da garganta

$L$ - comprimento do canal

$R$ - raio do duto

$R_{e q a n}-$ raio equivalente analítico

$Q^{*}$ - vazão considerando o raio equivalente ajustado

$R_{e q a j}$ - raio equivalente ajustado

\section{Símbolos Gregos}

$\tau$ - tensor tensão

$\mu$ - viscosidade absoluta

$\boldsymbol{\gamma}$ - taxa de deformação

$\beta$-constante no modelo macroscópico de fluido pseudo-plástico

$\rho$ - massa específica do fluido

$\dot{\gamma}$ - tensor taxa de deformação

$\eta$ - viscosidade

$\eta_{0}$ - viscosidade de baixas taxas de deformação

$\eta_{\infty}$ - viscosidade de altas taxas de deformação 
$\lambda$ - constante de tempo, normalmente chamada de tempo de relaxação

$\alpha-(3 n+1)$

$\nabla$-gradiente de um vetor

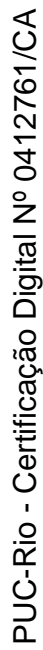

